\title{
SUSY-breaking scenarios with a mildly violated $R$ symmetry
}

\author{
Constantinos Pallis ${ }^{\mathrm{a}}$ \\ Laboratory of Physics, Faculty of Engineering, Aristotle University of Thessaloniki, 54124 Thessaloniki, Greece
}

Received: 1 September 2020 / Accepted: 28 August 2021 / Published online: 9 September 2021

(C) The Author(s) 2021

\begin{abstract}
New realizations of the gravity-mediated SUSY breaking are presented consistently with an $R$ symmetry. We employ monomial superpotential terms for the hidden-sector (goldstino) superfield and Kähler potentials parameterizing compact or non-compact Kähler manifolds. Their scalar curvature may be systematically related to the $R$ charge of the goldstino so that Minkowski solutions without fine tuning are achieved. A mild violation of the $R$ symmetry by a higher order term in the Kähler potentials allows for phenomenologically acceptable masses for the $R$ axion. In all cases, nonvanishing soft SUSY-breaking parameters are obtained and a solution to the $\mu$ problem of MSSM may be accommodated by conveniently applying the Giudice-Masiero mechanism.
\end{abstract}

\section{Introduction}

The fact that Supersymmetry (SUSY) remains still unobservable in the nature does not invalidate its importance in constructing theories beyond the Standard Model (SM). Most notably, the problem of SUSY breaking is currently under intense scrutiny - see e.g. Refs. [1-22] - and new settings are suggested within Supergravity (SUGRA) [23] aiming to obtain natural Minkowski and/or de Sitter vacua - the latter possibility is not so favored, though, because of the controversy $[24,25]$ surrounding this kind of (meta) stable vacua. A crucial question in this area of research concerns $[2,3]$ the specific forms of superpotential and the type of geometry which may be adopted.

Trying to reply to these questions in a recent paper [1], we employed as guideline an approximate $R$ symmetry $[23,27]$ which totally fixes the form of the superpotential, $W_{\mathrm{H}}$, as a function of the goldstino superfield, $Z,-$ contrary to the well-known and widely adopted Polonyi model [26] where the same $R$ symmetry is badly violated by a constant term in $W_{\mathrm{H}}$. We show that if we associate $W_{\mathrm{H}}$ with a Kähler

a e-mail: kpallis@gen.auth.gr (corresponding author) potential, $K_{\mathrm{H}}$, which parameterizes the $S U(1,1) / U(1)$ Kähler manifold with constant curvature $-1 / 2$ we can obtain a Minkowski vacuum (i.e., SUSY breaking with vanishing classical vacuum energy) without unnatural fine tuning, and non-vanishing soft SUSY-breaking (SSB) parameters [3436], of the order of the gravitino mass, $m_{3 / 2}$, which is readily determined at the tree level. A solution to the $\mu$ problem of Minimal Supersymmetric SM (MSSM) may be also achieved by suitably applying the Giudice-Masiero mechanism [37] for an updated review see Refs. [38,39]. The possibly problematic, from astrophysical point of view [40-44], (pseudo) Nambu-Goldstone boson named $R$ axion [33] may acquire acceptably large mass by mildly violating the $R$ symmetry in $K_{\mathrm{H}}$ without any impact either to the minimization of the SUGRA potential or to the values of the SSB parameters.

In the present paper, motivated by several related models $[2,3,20,45-47]$, we extend our approach above, considering different $R$ charges for $Z$ and allowing, thereby, for other (not only linear) monomial forms of $W_{\mathrm{H}}(Z)$ - see Sect. 2. We show that natural Minkowski solutions, similar to those found in Ref. [1], can be accommodated by conveniently selecting the geometry of the internal space. Contrary to Ref. [1], we here consider compact [28-31] and non-compact [2,3,32] Kähler manifolds, whose the curvature is determined as a simple function of the $R$ charge of $Z$. In both cases, we specify new SUSY-breaking minima and show that the same quartic term introduced in Refs. [1-3] provides mass for the $R$ axion without spoiling, though, the minimization of the SUGRA potential. Following the analysis of Ref. [1], we combine our hidden sectors with some sample observable ones and broaden our results as regards the SSB parameters and the derivation of the $\mu$ term of MSSM - see Sect. 3. Our conclusions are further discussed in Sect. 4. An alternative formulation of our set-up is presented in Appendix A.

Unless otherwise stated, we use units where the reduced Planck mass $m_{\mathrm{P}}=2.433 \cdot 10^{18} \mathrm{GeV}$ is taken to be unity and charge conjugation is denoted by a bar. 


\section{Hidden sector}

Here we first - in Sect. 2.1 - specify the hidden sector of our model and then - in Sect. 2.2 - investigate the SUSYbreaking mechanism employing the curvature of the Kähler manifold as free parameter. Lastly, in Sect. 2.3, we introduce the $R$-symmetry violating term and compute the mass of the $R$ axion.

\subsection{Model set-up}

Following our strategy in Ref. [1], we consider a hidden sector consisting of just one gauge-singlet superfield $Z$. The corresponding superpotential $W_{\mathrm{H}}$ is fixed by imposing an $R$ symmetry under which $Z$ has $R$ charge $2 / v-$ and not just 2 as in Ref. [1] - whereas that of $W_{\mathrm{H}}$ is taken to be 2, i.e.,

$W_{\mathrm{H}}=m Z^{v}$

where $m$ is a positive, free parameter with mass dimensions. Also $v \neq 0$ is an exponent which may, in principle, acquire any real value. This is possible if we consider $W_{\mathrm{H}}$ as an effective superpotential valid close to the non-zero vacuum of the theory. E.g., we can obtain $v<0$ from dynamical breaking of a $S U(N)$ continuous symmetry [48] assuming in addition [46] that this is not a gauge symmetry to avoid Dterm contributions in the SUGRA potential. Moreover, $v>0$ fractional numbers are already considered in Refs. [2,3,45] and, as discussed in Appendix A, support a complete form of $W_{\mathrm{H}}$ invoking an appropriate field redefinition [47].

Contrary to the Polonyi model [26] we do not consider any $R$-symmetry violating constant term in $W_{\mathrm{H}}$. Such a violation is accommodated in the Kähler potential which is

$K_{\mathrm{H}}=N \ln \left(1+\frac{|Z|^{2}-k Z_{-}^{4}}{N}\right)$

with $Z_{ \pm}=Z \pm \bar{Z}$. Here $N$ and $k$ are free parameters of either sign. Motivated by several superstring and D-brane models $[50,51]$ we consider the integer negative values of $N$ as the most natural. However, positive $N$ 's can not be disregarded if we limit our attention on SUGRA [28-31]. On the other hand, $k$ is a parameter which mildly violates $R$ symmetry. We call this violation "mild" since (a) $k$ is involved in a higher-order (non quadratic) term in $K_{\mathrm{H}}$ and (b) tiny $k$ values endow $R$ axion with phenomenologically acceptable masses - see Sect. 2.3. The presence of $k$ in $K_{\mathrm{H}}$ is totally natural, according to the argument [49] of 't Hooft, since nullifying it the $R$ symmetry becomes exact. In view of this fact, the positivity of the argument of logarithm in Eq. (2) implies

$1+|Z|^{2} / N \gtrsim 0 \Rightarrow-|Z|^{2} / N \lesssim 1$

which is true for any $N>0$ but dictates

$|Z|^{2} \lesssim-N$ for $N<0$
The curved space parameterized by $K_{\mathrm{H}}$ has metric

$\mathrm{g}:=\partial_{Z} \partial_{\bar{Z}} K_{\mathrm{H}}=N w\left(N+|Z|^{2}-k Z_{-}^{4}\right)^{-2}$

where we find it convenient to define

$w=N\left(1+12 k Z_{-}^{2}\right)+k Z_{-}^{2}\left(3 Z_{+}^{2}+4 k Z_{-}^{4}\right)$.

Substituting Eqs. (1) and (2) in the well-known formula

$V:=e^{G}\left(G^{I \bar{J}} G_{I} G_{\bar{J}}-3\right)$ with $G:=K_{\mathrm{H}}+\ln \left|W_{\mathrm{H}}\right|^{2}$,

$I, J=Z, G^{Z \bar{Z}}=\mathrm{g}^{-1}, G_{I}:=\partial_{I} G$ and $G_{\bar{J}}:=\partial_{\bar{J}} G$, we find the hidden-sector (F-term) scalar potential

$V_{\mathrm{H}}=\frac{m^{2}}{N} e^{K_{\mathrm{H}}}|Z|^{2(v-1)}\left(\frac{u v}{w}-3 N|Z|^{2}\right)$.

Here $w$ originates from the numerator in Eq. (5a) and we introduce the quantities

$u=N v+(N+v)|Z|^{2}-k Z_{-}^{3}\left(v Z_{-}+4 N Z\right)$,

$v=N v+(N+v)|Z|^{2}-k Z_{-}^{3}\left(v Z_{-}-4 N \bar{Z}\right)$,

which arise from the numerators of $G_{Z}$ and $G_{\bar{Z}}$ in Eq. (6). Written in this form, $V_{\mathrm{H}}$ may be directly compared to the one employed in Ref. [1].

\subsection{The $R$-symmetric limit}

If we set $k=0$ in Eq. (2) we obtain the exactly $R$-symmetric form of $K_{\mathrm{H}}, K_{\mathrm{H} 0}$, which parameterizes the coset spaces $S U(1,1) / U(1)$ for $N<0$ or $S U(2) / U(1)$ for $N>0$ [28], with metric and constant scalar curvature respectively

$\mathrm{g}^{(0)}=\partial_{Z} \partial_{\bar{Z}} K_{\mathrm{H} 0}=\left(1+\frac{|Z|^{2}}{N}\right)^{-2}$ and $\mathcal{R}_{\mathrm{H}}^{(0)}=\frac{2}{N}$.

The last quantity reveals that the Kähler manifold is compact or non-compact if $N>0$ or $N<0$. Here and hereafter, the superscript (0) and the subscript 0 denote quantities corresponding to the totally $R$-symmetric case. Working in this simplified framework, it is more convenient to investigate the existence of Minkowski solutions, as done in Sect. 2.2.1, classify the relevant solutions - see Sect. 2.2.2 - and derive the relevant particle spectrum in Sect. 2.2.3.

Let us clarify here that the $S U(1,1) / U(1)$ manifold is also parameterized by the half-plane coordinates (usually notated by $T$ and $\bar{T}$ ) which are related to the disc coordinates ( $Z$ and $\bar{Z})$ through a Cayley transform $[1,28,32]$. This parameterization, though, violates the $R$ symmetry and so it is inappropriate for our purposes. Note that the parameterization of the $S U(2) / U(1)$ space does not change under that transform. 


\subsubsection{Minkowski vacua}

Substituting $k=0$ into Eq. (7) and taking into account that

$G_{Z}^{(0)}=\bar{G}_{\bar{Z}}^{(0)}=\frac{1}{Z}\left(\sqrt{\mathrm{g}^{(0)}}|Z|^{2}+v\right)$.

we derive the corresponding SUGRA potential

$V_{\mathrm{H} 0}=\frac{m^{2}}{N^{2}} \frac{e^{K_{\mathrm{H} 0}}}{|Z|^{2(1-v)}}\left(\left(v N+(N+v)|Z|^{2}\right)^{2}-3 N^{2}|Z|^{2}\right)$,

which, as expected, depends exclusively on $|Z|^{2}$. The systematic search of a Minkowski vacuum - defined by the simultaneous fulfilment of the conditions
(a) $\left\langle V_{\mathrm{H} 0}\right\rangle=0$,
(b) $\left\langle V_{\mathrm{H} 0}^{\prime}\right\rangle=0$ and
(c) $\left\langle V_{\mathrm{H} 0}^{\prime \prime}\right\rangle>0$,

where the derivatives with respect to (w.r.t) $|Z|^{2}$ are denoted by a prime - is facilitated if the expression in the parenthesis of Eq. (11) is expanded as follows

$$
(2 v(N+v)-3 N) N|Z|^{2}+v^{2} N^{2}+|Z|^{4}(N+v)^{2} .
$$

From our experience in Ref. [1], we suspect that the attainment of a Minkowski vacuum is, in principle, possible if the expression in Eq. (13) equals to a perfect square trinomial including a minus in the double product. Since the last two terms consist a sum of squares, the desired perfect square trinomial is achieved, if the two first terms in Eq. (13) coincide with the minus double product of the square root of anyone of the two last terms. More explicitly, we impose the condition

$-2|N||v||N+v|=(2 v(N+v)-3 N) N$

which, fortunately, is independent of $|Z|^{2}$. We seek below the $N=N_{\mathrm{c}}$ values as a function of $v$ that satisfy the condition above. It is obvious that we need a combination of $v, N_{\mathrm{c}}$ and $N_{\mathrm{c}}+v$ such that $-2\left|N_{\mathrm{c}}\|v\| N_{\mathrm{c}}+v\right|=-2 N_{\mathrm{c}} v\left(N_{\mathrm{c}}+v\right)$ since, otherwise, this term is cancelled out in Eq. (14) and the only possible solution is $N_{\mathrm{c}}=0$. As a consequence, we single out the following cases which yield $N_{\mathrm{c}} \neq 0$ :

(i) $v>0$ and $N_{\mathrm{c}}>0$ resulting to $v+N_{\mathrm{c}}>0$;

(ii) $v<0, N_{\mathrm{c}}>0$ and $v+N_{\mathrm{c}}<0$;

(iii) $v>0, N_{\mathrm{c}}<0$ and $v+N_{\mathrm{c}}<0$.

If we solve Eq. (14) w.r.t $N_{\mathrm{c}}$, in all cases above, we can derive $N_{\mathrm{c}}$ using $v$ as free variable. Namely,

$N_{\mathrm{c}}=\frac{4 v^{2}}{3-4 v} \Rightarrow N_{\mathrm{c}} \gtrless 0$ for $v \lessgtr 3 / 4$.

Turning the argument around, we can obtain two possible solutions $\nu_{ \pm}$for every (preferably integer) $N_{\mathrm{c}}$ value if we solve Eq. (15) w.r.t v, i.e.,

$\nu_{ \pm}=\frac{1}{2}\left(-N_{\mathrm{c}} \pm \sqrt{N_{\mathrm{c}}\left(N_{\mathrm{c}}+3\right)}\right)$

and correspond to real numbers if

$N_{\mathrm{c}}\left(N_{\mathrm{c}}+3\right) \geq 0 \Rightarrow N_{\mathrm{c}} \geq 0$ or $\quad N_{\mathrm{c}} \leq-3$.

For $N=N_{\mathrm{c}}, V_{\mathrm{H} 0}$ in Eq. (11) includes by construction the square of a binomial with a minus inside. Indeed, it reads

$$
V_{\mathrm{H} 0 \mathrm{c}}=\left(\frac{m}{4 v}\right)^{2}\left(1+\frac{|Z|^{2}}{N_{\mathrm{c}}}\right)^{N_{\mathrm{c}}}|Z|^{2(v-1)}\left(3|Z|^{2}-4 v^{2}\right)^{2} .
$$

Here and hereafter the subscript "c" denotes quantities computed for $N=N_{\mathrm{c}}$. Enforcing Eq. (12b) we find a honest extremum of $V_{\mathrm{H} 0 \mathrm{c}}$ as follows

$$
\left\langle V_{\mathrm{H} 0 \mathrm{c}}^{\prime}\right\rangle=0 \Rightarrow\left\langle|Z|^{2}\right\rangle=4|\nu|^{2} / 3
$$

This result is, initially, restricted to $v \leq 3 / 2$ for $N_{\mathrm{c}}<0$ by virtue of Eqs. (4) and (17). It can be further constrained from the observation that Eq. (12a) is fulfilled for

$N_{\mathrm{c}} \neq-3 \Leftrightarrow v \neq 3 / 2$.

Indeed, taking $N_{\mathrm{c}}=-3$ and $v=3 / 2, V_{\mathrm{H} 0 \mathrm{c}}$ in Eq. (18) is simplified as

$V_{\mathrm{H} 0 \mathrm{c}}=27 m^{2} \sqrt{|Z|^{2}} /\left(12-4|Z|^{2}\right)$,

which exhibits just a SUSY vacuum. Finally, in order to check the validity of Eq. (12c), we compute

$$
\left\langle V_{\mathrm{H} 0 \mathrm{c}}^{\prime \prime}\right\rangle=2^{2 v-5} 3^{3-v}|v|^{2(v-1)} m^{2}\langle\mathrm{~g}\rangle^{-N_{\mathrm{c}} / 2},
$$

where we introduce the quantity

$$
\langle\mathrm{g}\rangle=\left\langle\mathrm{g}^{(0)}\right\rangle=\frac{1}{4}\left(1-\frac{2}{3} \mathrm{v}\right)^{-2}=\frac{9 N_{\mathrm{c}}}{16 v^{2}\left(N_{\mathrm{c}}+3\right)},
$$

which controls the stability of the vacuum in Eq. (19) - as we show below, even for $k \neq 0$ in Eq. (2) the Minkowski vacuum lies along the direction $Z=Z_{-}$and so we do not apply the symbolic distinction mentioned below Eq. (9) regarding $\langle\mathrm{g}\rangle$ and $\left\langle\mathrm{g}^{(0)}\right\rangle$. Namely, $v<3 / 2$ assures the validity of Eq. (12c) for any $N_{\mathrm{c}}$. Taking into account also, the constraint below Eq. (19) for $N_{\mathrm{c}}<0$ and Eqs. (15) and (17) we end up with the following allowed domains

$\frac{3}{4}<v<\frac{3}{2}$ for $N_{\mathrm{c}}<0$ and $v<\frac{3}{4}$ for $N_{\mathrm{c}}>0$.

Note that the $v$ range is rather limited for $N_{\mathrm{c}}<0$ and ampler for $N_{\mathrm{c}}>0$. 
Table 1 Solutions to Eq. (15) for various v's

\begin{tabular}{lllllll}
\hline$\nu_{-}$ & $5 / 4$ & $6 / 5$ & 1 & $7 / 8$ & $5 / 6$ & $4 / 5$ \\
$N_{\mathrm{c}}$ & $-25 / 8$ & $-16 / 5$ & -4 & $-49 / 8$ & $-25 / 3$ & $-64 / 5$ \\
\hline$\nu_{-}$ & $-1 / 2$ & -1 & $-3 / 2$ & -2 & $-5 / 2$ & -3 \\
$N_{\mathrm{c}}$ & $1 / 5$ & $4 / 7$ & 1 & $16 / 11$ & $25 / 13$ & $12 / 5$ \\
\hline
\end{tabular}

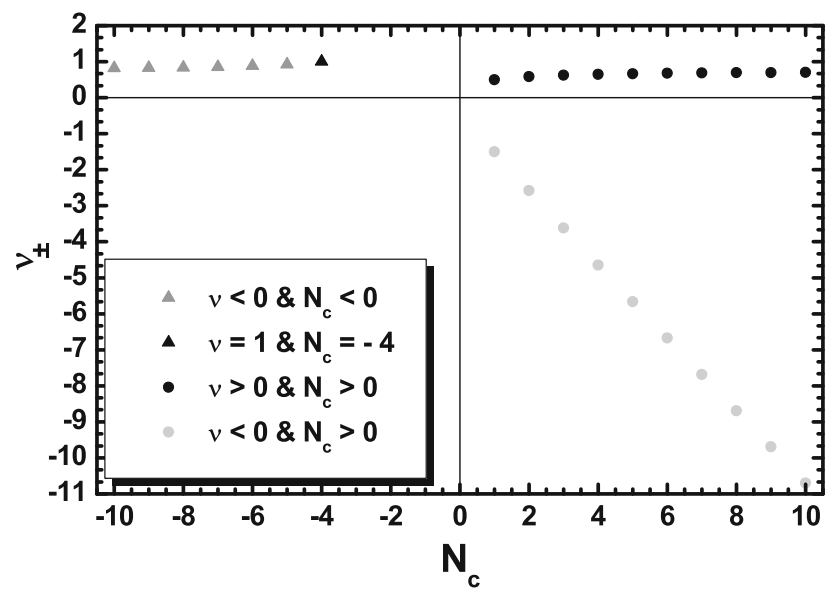

Fig. 1 Solutions to Eq. (16) for integer $N_{\mathrm{c}}$ values and $\left|N_{\mathrm{c}}\right| \leq 10$. We take $N_{\mathrm{c}}<0$ (gray and black triangles), or $N_{\mathrm{c}}>0$ and $v>0$ (black circles) or $N_{\mathrm{c}}>0$ and $v<0$ (light gray circles)

\subsubsection{Classification of the solutions}

To be more specific, we list in Table 1 some pairs $\left(v, N_{\mathrm{c}}\right)$ compatible with Eqs. (15) and (24). All of these correspond to $v=v_{-}$in Eq. (16) and yield integer $N_{\mathrm{c}}$ for $v=1$ and $-3 / 2$. Confining ourselves on exclusively integer $N_{\mathrm{c}}$ - favored by the string theories $[50,51]-$, we may vary $N_{\mathrm{c}}$ in the range $[-10,10]$ and obtain $v$ via Eq. (16). We depict the results in Fig. 1, where we present three families of points of different shapes corresponding to different types of $V_{\mathrm{H} 0 \mathrm{c}}$ as we explain below. Besides the $v$ values corresponding to $N_{\mathrm{c}}=-4$ and 1 , the others are irrational numbers which, are used mainly for presentational purposes - see, e.g., Refs. [2,3]. Also, for $N_{\mathrm{c}}>0$ both outputs, $v_{+}$and $v_{-}$, are possible and, in particular, the $v_{-}$values increase almost linearly with $N_{\mathrm{c}}$ whereas the $v_{+}$values remain almost unchanged at the level of the values obtained for $N_{\mathrm{c}}<0$.

The structure of $V_{\mathrm{HOc}}$ in Eq. (18) for the various pairs $\left(v, N_{\mathrm{c}}\right)$ satisfying Eq. (15) can be inferred by Figs. 2 and 3, where we present $V_{\mathrm{H} 0 \mathrm{c}} / m^{2} m_{\mathrm{P}}^{2}$ as a function of $|Z|^{2}$ for $N_{\mathrm{c}}<$ 0 - in Fig. 2 - and for $N_{\mathrm{c}}>0$ - in Fig. 3. Namely, we draw solid, dashed and dot-dashed lines for $\left(v_{-}, N_{\mathrm{c}}\right)=(1,-4)$, $(4 / 5,-64 / 5)$ and $(5 / 4,-25 / 8)$ respectively in Fig. 2 . On the other hand, the dashed, solid and dot-dashed lines in Fig. 3 are obtained for $\left(v_{+}, N_{\mathrm{c}}\right)=(1 / 2,1),\left(v_{-}, N_{\mathrm{c}}\right)=(-3 / 2,1)$ and $\left(v_{-}, N_{\mathrm{c}}\right)=(-2,16 / 11)$ respectively. The line $V_{\mathrm{H} 0 \mathrm{c}}=0$ and the values $\left\langle|Z|^{2}\right\rangle$ at the non-SUSY minimum - see Eq. (19) -

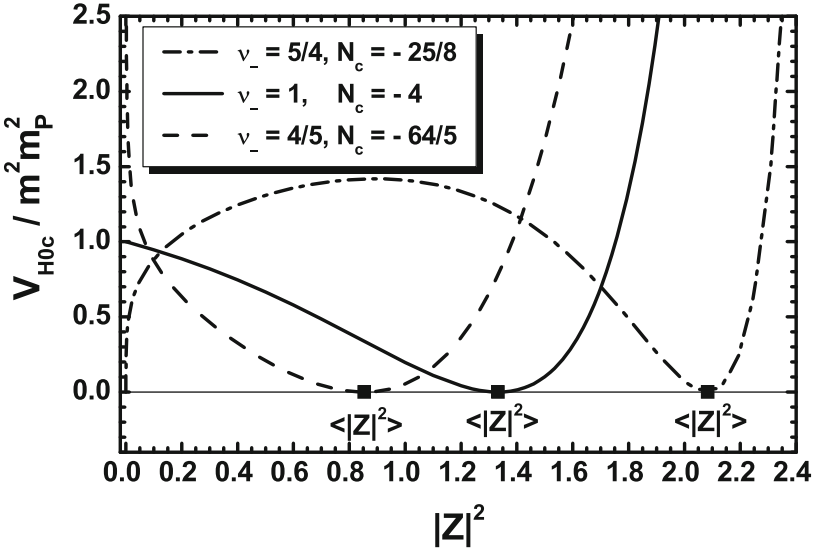

Fig. 2 The (dimensionless) hidden-sector potential $V_{\mathrm{H} 0 \mathrm{c}} / m^{2} m_{\mathrm{P}}^{2}$ in Eq. (11) as a function of $|Z|^{2}$ for $\left(v_{-}, N_{\mathrm{c}}\right)=(4 / 5,-64 / 5),(1,-4)$ and $(5 / 4,-25 / 8)$ (dashed, solid and dot-dashed line respectively). The line $V_{\mathrm{H} 0 \mathrm{c}}=0$ and the values $\left\langle|Z|^{2}\right\rangle$ are also indicated

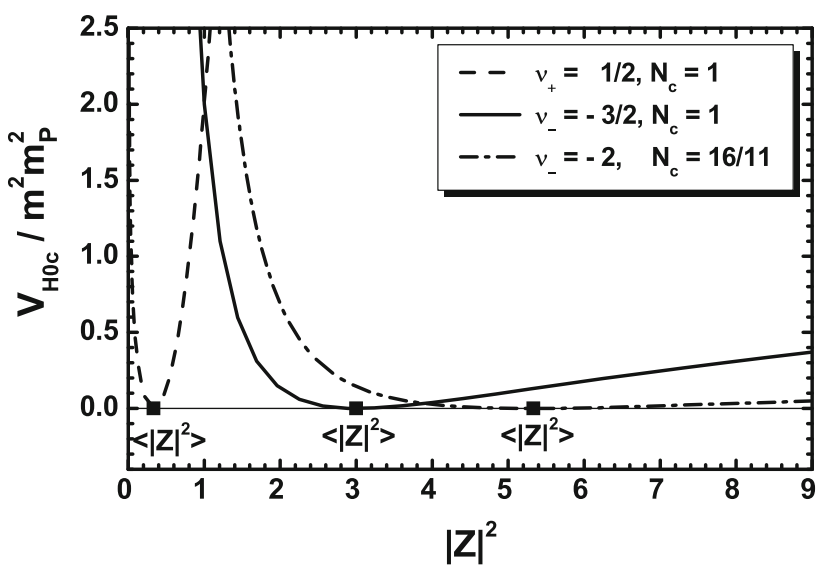

Fig. 3 The same as in Fig. 2 but for $\left(v, N_{\mathrm{c}}\right)=(1 / 2,1),(-3 / 2,1)$, and $(-2,16 / 11)$ (dashed, solid and dot-dashed line respectively)

Table 2 Minkowski vacua for the $v$ values used in Figs. 2 and 3

\begin{tabular}{lllllll}
\hline & Figure 2 & \multicolumn{5}{c}{ Figure 3 } \\
\hline$v$ & $5 / 4$ & 1 & $4 / 5$ & -2 & $-3 / 2$ & $1 / 2$ \\
$\left\langle|Z|^{2}\right\rangle$ & $25 / 12$ & $4 / 3$ & $64 / 75$ & $16 / 3$ & 3 & $1 / 3$ \\
\hline
\end{tabular}

are also indicated in both figures. More explicitly, the specific values of $\left\langle|Z|^{2}\right\rangle$ for each $v$ value employed in the figures above are arranged in Table 2 . We see that $\left\langle|Z|^{2}\right\rangle$ decreases with $|v|$ and may become even subplanckian, in sharp contrast to the well-known Polonyi model [26] and the case of Ref. [1].

Let's focus first on Fig. 2. We remark that for $v>1$ in the domain of Eq. (24), $V_{\mathrm{H} 0 \mathrm{c}}$ features a SUSY minimum at $\langle|Z|\rangle=0$ apart from the non-SUSY Minkowski one - see dot-dashed line in Fig. 2. This is due to the presence of the factor $|Z|^{2(v-1)}$ in Eq. (11) which yields another solution to the right equation in Eq. (19) for $v>1$. Although less attractive than the other cases, the coexistence of SUSY and 
non-SUSY Minkowski vacua may be beneficial for applying the multi-point principle analyzed in Ref. [20]. We obtain the same shape of $V_{\mathrm{H} 0 \mathrm{c}}$ for $1<v<3 / 2$. Since no integer $N_{\mathrm{c}}$ value may be computed via Eq. (15) for $v$ values into this domain, no point in Fig. 1 corresponds to this kind of $V_{\mathrm{H} 0 \mathrm{c}}$. For the value $\left(v, N_{\mathrm{c}}\right)=(1,-4)$ - black triangle in Fig. 1 - we obtain the well-known form of $V_{\mathrm{HOc}}$ presented in Ref. [1], whereas for the remaining (gray) triangles in Fig. 1 we obtain the type of $V_{\mathrm{H} 0 \mathrm{c}}$ drawn by dashed line in Fig. 2. It is remarkable that $V_{\mathrm{H} 0 \mathrm{c}}$ develops just one critical point in this case. The situation is rather different for $N_{\mathrm{c}}>0$ as shown in Fig. 3. For $v>0-$ e.g., see the $\left(v, N_{\mathrm{c}}\right)$ values represented by black circles in Fig. 1 -, the type of $V_{\mathrm{H} 0 \mathrm{c}}$ corresponds to the dashed line in Fig. 3. On the contrary, the $v<0$ values give rise to $V_{\mathrm{H} 0 \mathrm{c}}$ whose structure is designed by the solid and the dot-dashed lines. As $N_{\mathrm{c}}$ increases, $\left|N_{\mathrm{c}}\right|$ increases too - see e.g., the gray circles in Fig. $1-$ and $\left\langle|Z|^{2}\right\rangle$ is moved towards larger values.

\subsubsection{Particle spectrum}

If we analyze $Z$ according to the description

$Z=(z+i \theta) / \sqrt{2}$

and expand $V_{\mathrm{H} 0 \mathrm{c}}$ in Eq. (18) about the configuration

$\langle z\rangle=2 \sqrt{\frac{2}{3}}|\nu|$ and $\langle\theta\rangle=0$,

- cf. Eq. (19) -, we can work out the hidden-sector spectrum of the model. This is composed of:

(i) A (canonically normalized) real scalar field - the sgolstino or $R$ saxion,$- \widehat{z}=\sqrt{\langle\mathrm{g}\rangle} z$, with mass squared

$m_{\widehat{z}}^{2}=\left\langle\partial_{\bar{z}}^{2} V_{\mathrm{H} 0 \mathrm{c}}\right\rangle=\left\langle 2 V_{\mathrm{H} 0 \mathrm{c}}^{\prime \prime}|Z|^{2} / \mathrm{g}\right\rangle=\frac{9}{4} \frac{m_{3 / 2}^{2}}{|\nu|^{2}\langle\mathrm{~g}\rangle} ;$

where we take into account Eqs. (22) and (23),

(ii) The gravitino, $\widetilde{G}$, - which absorbs the fermionic partner of the $R$ saxion, the $R$ axino - with mass squared

$$
m_{3 / 2}^{2}=m^{2}\left\langle e^{K_{\mathrm{Hc}}}|Z|^{2 v}\right\rangle=4^{v}|v|^{2 v} m^{2} / 3^{v}\langle\mathrm{~g}\rangle^{N_{\mathrm{c}} / 2}
$$

(iii) A massless Nambu-Goldstone boson, $\theta$, - referred [33] to as an $R$ axion.

Besides the last one, the masses above keep their values even for $k \neq 0$ and obey the super-trace formula [23] which, in the case of an hidden sector with one superfield, reads [1]

$$
\mathrm{STr} M_{0}^{2}=m_{\widehat{z}}^{2}-4 m_{3 / 2}^{2}=6 m_{3 / 2}^{2}\left\langle\mathcal{R}_{\mathrm{Hc}}^{(0)}\right\rangle=\frac{12}{N_{\mathrm{c}}} m_{3 / 2}^{2},
$$

where we make use of Eqs. (9), (15), (27b) and (27a).
2.3 Including the $R$-symmetry-violating term

The (canonically normalized) $R$ axion, $\widehat{\theta}$, couples to the massless SM gauge bosons (gluons and photons) [53-56] due to $S U(3)_{\text {c }}$ and $U(1)_{\mathrm{EM}}$ anomalies respectively. Nonperturbative $S U(3)_{\text {c }}$ instanton effects [52] result in a mass for $\widehat{\theta}$ which is expected to be at the $\mathrm{KeV}$ level and so it is severely constrained by astrophysical and laboratory probes. Here we focus on the most robust, lower bound derived from the fact that $\widehat{\theta}$ can be produced in the supernovae core via the bremsstrahlung process $n n \rightarrow n n \widehat{\theta}$ with $n$ being nucleons. The measurements of the neutrino burst from supernova 1987a set important constraints on sub-GeV axion-like particles. In particular, the energy loss rate due those particle should not exceed the one through neutrinos. Considering that the coupling between $\widehat{\theta}$ and gluons or photons is well suppressed, the restriction above is translated to the following bound on the mass of $\widehat{\theta}[40-44]$

$m_{\widehat{\theta}} \geq 10 \mathrm{MeV}$,

where we restore the units for convenience. On the other hand, we do not impose here an upper limit on $m_{\widehat{\theta}}$ from a possible candidacy of $\widehat{\theta}$ for cold dark matter since this depends crucially on the cosmological evolution of $\widehat{\theta}$ which is not specified in our set-up [53,54].

The phenomenological problems arising from the masslessness (at perturbative level) of $\widehat{\theta}$ for $k=0$ in Eq. (2) may be evaded invoking several modifications of the setting in Sect. 2.2. E.g., if we consider $Z$ as a nilpotent superfield [58] no sgoldstino appears in the particle spectrum and so no $R$ axion too. Another way out of this problem is the gauging of the $R$ symmetry [21,45,57], provided that it is anomalyfree. In this case, though, D-term contributions are introduced which destabilize the construction of Sect. 2.2.2. Following Ref. [1], we here adopt the simplest solution: we explicitly break the $R$ symmetry via a subdominant quartic term in $K_{\mathrm{H}}$ proportional to $k$ - see Eq. (2). As we show below, even for $k \neq 0$, the field configuration in Eq. (26) still corresponds to a Minkowski vacuum whereas a large enough mass for the $R$ axion is generated. This is actually the reason for which we prefer to activate the $R$-violation in $K_{\mathrm{H}}$ and not in $W_{\mathrm{H}}$ as in the Polonyi model [26]. It is clear that no purely theoretical motivation exists for this term in $K_{\mathrm{H}}$. Its presence, though, is "technically natural" thanks to the smallness of $k$ - see below. Although not generalized here, the exponent, 4, of this term is the unique which offers the "facilities" above [1]. The same term is widely utilized for the stabilization of the imaginary direction of the sgoldstino within the no-scale models [2,3].

Below we verify that $(\langle z\rangle,\langle\theta\rangle)$ defined in Eq. (26) represents a stable Minkowski vacuum of $V_{\mathrm{H}}$ in Eq. (7) for $k \neq 0$ and $N=N_{\mathrm{c}}$. To embark on it, we express $V_{\mathrm{H}}$ as a function of $z$ and $\theta$ using the parametrization in Eq. (25). First of all, 
we show that $V_{\mathrm{Hc}}$ at the point given by Eq. (26) vanishes, i.e.,

$$
\left\langle V_{\mathrm{Hc}}\right\rangle=0 \Rightarrow\left\langle u_{\mathrm{c}} v_{\mathrm{c}} / w_{\mathrm{c}}\right\rangle=3 N_{\mathrm{c}}\left\langle|Z|^{2}\right\rangle \text {. }
$$

Indeed, Eqs. (8a), (8b) and (5b) at the vacuum yield

$$
\left\langle u_{\mathrm{c}}\right\rangle=\left\langle v_{\mathrm{c}}\right\rangle=v N_{\mathrm{c}}+\left(N_{\mathrm{c}}+v\right)\left\langle|Z|^{2}\right\rangle \text { and }\left\langle w_{\mathrm{c}}\right\rangle=N_{\mathrm{c}} \text {, }
$$

and so Eq. (30) can be readily deduced. Then we minimize $V_{\mathrm{Hc}}$ w.r.t $z$ and $\theta$. Given that $Z_{-}=\sqrt{2} i \theta, V_{\mathrm{Hc}}$ for $\theta=0$ coincides with the one in Eq. (18), i.e.,

$V_{\mathrm{Hc}}(z, \theta=0)=V_{\mathrm{H} 0 \mathrm{c}}$

and therefore, $\langle z\rangle$ (for $k \neq 0$ ) keeps its value in Eq. (26) (found for $k=0$ ). As regards the $\theta$ direction, we check the validity of the extremum condition computing the first derivative of $V_{\mathrm{Hc}}$ w.r.t $\theta$ for $\langle\theta\rangle=0$ with result

$$
\begin{aligned}
\left\langle\partial_{\theta} V_{\mathrm{Hc}}\right\rangle= & 3 m^{2}\left\langle e^{K_{\mathrm{Hc}}}|Z|^{2(v-1)}\right\rangle . \\
& \times\left\langle\left(\frac{\partial_{\theta} u_{\mathrm{c}}}{u_{\mathrm{c}}}+\frac{\partial_{\theta} v_{\mathrm{c}}}{v_{\mathrm{c}}}-\frac{\partial_{\theta} w_{\mathrm{c}}}{w_{\mathrm{c}}}\right)|Z|^{2}-\theta\right\rangle=0 .
\end{aligned}
$$

Here we take advantage of Eq. (30). We also observe that

$$
\left\langle\partial_{\theta}\left(e^{K_{\mathrm{H}}}|Z|^{2(v-1)}\right)\right\rangle=0
$$

since this is proportional at least to $\theta^{2}$ and, similarly,

$$
\left\langle\partial_{\theta} w_{\mathrm{c}}\right\rangle=\left\langle\partial_{\theta} u_{\mathrm{c}}\right\rangle=\left\langle\partial_{\theta} v_{\mathrm{c}}\right\rangle=0
$$

since these are proportional to $\theta$ - see Eqs. (5b), (8a) and (8b).

From the results above, it is easy to compute the mixed second derivatives

$$
\left\langle\partial_{z} \partial_{\theta} V_{\mathrm{Hc}}\right\rangle=\left\langle\partial_{\theta} \partial_{z} V_{\mathrm{Hc}}\right\rangle=0
$$

which are also zeroed. Therefore, the matrix of the second derivatives of $V_{\mathrm{H}}$ w.r.t $z$ and $\theta,\left\langle\partial_{i} \partial_{j} V_{\mathrm{Hc}}\right\rangle$ with $i=z$ and $\theta$ has a diagonal form. For $i=j=z$ we obtain the mass squared of $\widehat{z}$, given by Eq. (27a), since the $z$-dependent form of $V_{\mathrm{H}}$ in Eq. (7) coincides with that of $V_{\mathrm{H} 0 \mathrm{c}}$ in Eq. (18). For $i=j=\theta$, we differentiate once more $w_{\mathrm{c}}, u_{\mathrm{c}}$ and $v_{\mathrm{c}}$ w.r.t $\theta$ with results

$$
\begin{gathered}
\left\langle\partial_{\theta}^{2} w_{\mathrm{c}}\right\rangle=-48 k\left(\left\langle|Z|^{2}\right\rangle+N_{\mathrm{c}}\right), \\
\left\langle\partial_{\theta}^{2} u_{\mathrm{c}}\right\rangle=\left\langle\partial_{\theta}^{2} v_{\mathrm{c}}\right\rangle=\left(v+N_{\mathrm{c}}\right) .
\end{gathered}
$$

Armed with the results above we arrive at the following one,

$$
\begin{aligned}
\left\langle\partial_{\theta}^{2} V_{\mathrm{Hc}}\right\rangle= & 3 m^{2}\left\langle e^{K_{\mathrm{Hc}}}|Z|^{2(v-1)}\right\rangle \\
& \times\left(\left\langle|Z|^{2}\right\rangle\left\langle\frac{\partial_{\theta}^{2} u_{\mathrm{c}}}{u_{\mathrm{c}}}+\frac{\partial_{\theta}^{2} v_{\mathrm{c}}}{v_{\mathrm{c}}}-\frac{\partial_{\theta}^{2} w_{\mathrm{c}}}{w_{\mathrm{c}}}\right\rangle-1\right)
\end{aligned}
$$

Making use of the identity

$$
\left\langle|Z|^{2}\right\rangle\left(\frac{\partial_{\theta}^{2} u_{\mathrm{c}}}{u_{\mathrm{c}}}+\frac{\partial_{\theta}^{2} v_{\mathrm{c}}}{v_{\mathrm{c}}}\right)=1
$$

and canonically normalizing the relevant mode, we may achieve our final result

$m_{\widehat{\theta}}^{2}=\left\langle\partial_{\widehat{\theta}}^{2} V_{\mathrm{Hc}}\right\rangle=\left\langle\partial_{\theta}^{2} V_{\mathrm{Hc}} / \mathrm{g}\right\rangle=144 k m_{3 / 2}^{2} /\langle\mathrm{g}\rangle^{3 / 2}$,

which coincides with the one obtained in Ref. [1] for $v=1$. We observe that $m_{\widehat{\theta}}^{2}>0$ for $k>0$. Consequently, setting $k>0$ in Eq. (2) does not modify $V_{\mathrm{H}}$ from $V_{\mathrm{H} 0 \mathrm{c}}$ in the real direction but just allows for a non-vanishing $R$-axion mass. To fulfill Eq. (29) for, e.g., $m=1 \mathrm{TeV}$, it is enough to take $k \gtrsim 4 \cdot 10^{-13}$ - this number corrects a typo in Ref. [1] - for $v=v_{+}>0$ and $k \gtrsim 2 \cdot 10^{-14}-3 \cdot 10^{-5}$ for $v=v_{-}<0$, where the minimal $k$ increases as $N_{\mathrm{c}}$ increases from 1 to 10 - see Fig. 2. Therefore, our argument related to the naturalness of the $k$ term in Eq. (2) is compatible with the $R$-axion phenomenology.

Summarizing, the particle spectrum of the present version of our model comprises $\widetilde{G}, \widehat{z}$ and $\widehat{\theta}$ whose the masses are given by Eqs. (27b), (27a) and (39), respectively. We can verify that these masses obey the supertrace relation - cf. Eq. (28)

$$
\begin{aligned}
\mathrm{STr} M^{2} & =m_{\widehat{z}}^{2}+m_{\widehat{\theta}}^{2}-4 m_{3 / 2}^{2}=6 m_{3 / 2}\left\langle\mathcal{R}_{\mathrm{Hc}}\right\rangle \\
& =\frac{9}{v}\left(\frac{1}{v}-\frac{4}{3}\left(1-12 k v /\langle\mathrm{g}\rangle^{3 / 2}\right)\right) m_{3 / 2}^{2},
\end{aligned}
$$

where $\left\langle\mathcal{R}_{\mathrm{Hc}}\right\rangle$ is geometrically [1] estimated as

$$
\left\langle\mathcal{R}_{\mathrm{Hc}}\right\rangle=\left\langle\mathcal{R}_{\mathrm{Hc}}^{(0)}\right\rangle-24 k /\langle\mathrm{g}\rangle^{3 / 2},
$$

with $\left\langle\mathcal{R}_{\mathrm{Hc}}^{(0)}\right\rangle$ given in Eq. (9). Contrary to the case with $k=0$, here $\mathcal{R}_{\mathrm{Hc}}$ is not generically constant.

Checking the hierarchy of the various masses, we infer from Eqs. (27b) and (27a) that

$m_{3 / 2} \leq m_{\widehat{z}}$ for $v\langle\mathrm{~g}\rangle^{1 / 2} \leq 3 / 2$

and from Eqs. (27b) and (39) that

$m_{\widehat{\theta}} \leq m_{3 / 2}$ for $k \leq\langle\mathrm{g}\rangle^{3 / 2} / 144$. 


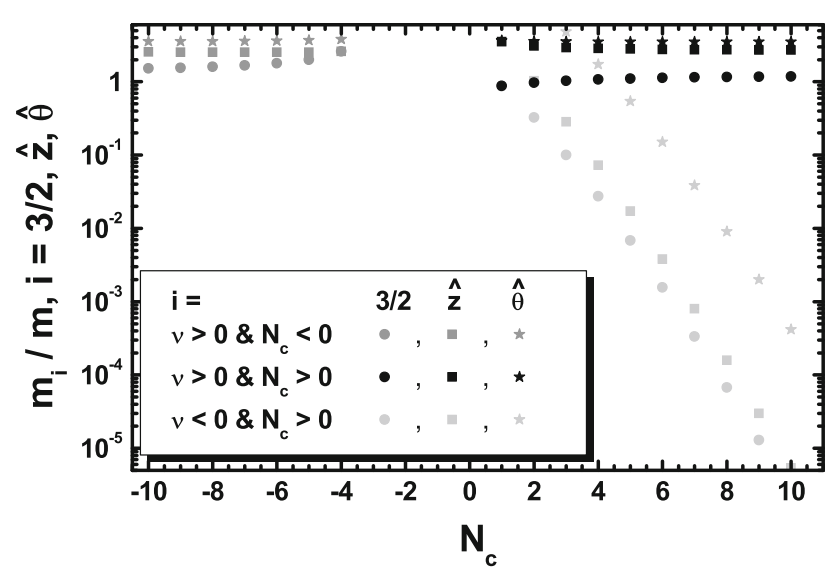

Fig. 4 The ratios $m_{3 / 2} / m, m_{\widehat{z}} / m$ and $m_{\hat{\theta}} / m$ for $k=0.05$ (circles, squares and stars respectively) versus $N_{\mathrm{c}}$. We take $N_{\mathrm{c}}<0$ (gray symbols) or $N_{\mathrm{c}}>0$ and $v>0$ (black symbols) or $N_{\mathrm{c}}>0$ and $v<0$ (light gray symbols)

More specifically, we obtain $m_{\widehat{z}} \leq 2 m_{3 / 2}$ for $v>0$ and $N_{\mathrm{c}}<0$ and so the decay of $\widehat{z}$ into a pair of $\widetilde{G}[53,54]$ is kinematically blocked whereas for $N_{\mathrm{c}}>0$ this decay channel is open. Therefore, the decay of $\widehat{z}$ and $\widehat{\theta}$ into $\widetilde{G}$ depends on the parameters ( $v, N_{\mathrm{c}}$ and $k$ ) and may have an impact on the relic abundance of $\widetilde{G}$ before nucleosynthesis.

These conclusions are illustrated in Fig. 4, where we display the ratios $m_{3 / 2} / m$ (circles), $m_{\widehat{z}} / m$ (squares) and $m_{\widehat{\theta}} / m$ (stars) for $k=0.05$ versus $N_{\mathrm{c}}$, for integer $N_{\mathrm{c}}$ values and $\left|N_{\mathrm{c}}\right| \leq 10$. We take $N_{\mathrm{c}}<0$ (gray symbols) or $N_{\mathrm{c}}>0$ and $v>0$ (black symbols) or $N_{\mathrm{c}}>0$ and $v<0$ (light gray symbols). For the selected $k$ value $\widehat{\theta}$ is heavier than both $\widehat{z}$ and $\widetilde{G}$ for any $\left(\nu, N_{\mathrm{c}}\right)$ whereas the hierarchy of $\widehat{z}$ and $\widetilde{G}$ is $k$-independent and mildly dependents on $\left(v, N_{\mathrm{c}}\right)$ according to Eq. (42a).

Just for completeness, we mention that the $R$ axion may acquire mass squared

$m_{\widehat{\theta}}^{2}=144 k m_{3 / 2}^{2} /\langle\mathrm{g}\rangle^{2}$

similar to the one in Eq. (39), if we adopt one of the following Kähler potentials

$K_{\mathrm{H}}=N_{\mathrm{c}} \ln \left(1+|Z|^{2} / N_{\mathrm{c}}\right)+N_{k} \ln \left(1-k Z_{-}^{4} / N_{k}\right)$,

$K_{\mathrm{H}}=N_{\mathrm{c}} \ln \left(1+|Z|^{2} / N_{\mathrm{c}}\right)-k Z_{-}^{4}$,

where $N_{k}$ is an undetermined constant.

\section{Observable sector}

Our next task is to study the transmission of the SUSY breaking to the visible world. To implement this, we introduce the chiral superfields of the observable sector $\Phi_{\alpha}$ and assume the following structure - cf. Refs. [1,26] - for the total superpotential, $W$, and Kähler potential, $K$, of the theory

$W=W_{\mathrm{H}}(Z)+W_{\mathrm{O}}\left(\Phi_{\alpha}\right)$,

$K=K_{\mathrm{Hc}}(Z)+\widetilde{K}(Z)\left|\Phi_{\alpha}\right|^{2}$,

where $W_{\mathrm{H}}$ and $K_{\mathrm{Hc}}$ are given by Eqs. (1) and (2) with $N=N_{\mathrm{c}}$ whereas $W_{\mathrm{O}}$ and $\widetilde{K}$ are specified in Sect. 3.1 for a generic SUSY model and, in Sect. 3.2, for the MSSM. In the latter case, a solution to the $\mu$ problem is also proposed.

\subsection{Generic model}

Here we adopt the following quite generic form of $W_{\mathrm{O}}$

$W_{\mathrm{O}}=h \Phi_{1} \Phi_{2} \Phi_{3}+\mu \Phi_{4} \Phi_{5}$

where we may easily select the appropriate $R$ charge for each of $\Phi_{\alpha}$ as in Ref. [1]. We try also similar $\widetilde{K}$, ensuring universal SSB parameters for $\Phi_{\alpha}$, i.e.,

$$
\begin{aligned}
& K_{1}=K_{\mathrm{Hc}}+\sum_{\alpha}\left|\Phi_{\alpha}\right|^{2}, \\
& K_{2}=N_{\mathrm{c}} \ln \left(1+\left(|Z|^{2}-k Z_{-}^{4}+\sum_{\alpha}\left|\Phi_{\alpha}\right|^{2}\right) / N_{\mathrm{c}}\right),
\end{aligned}
$$

$K_{3}=K_{\mathrm{Hc}}+N_{\mathrm{O}} \ln \left(1+\sum_{\alpha}\left|\Phi_{\alpha}\right|^{2} / N_{\mathrm{O}}\right)$

where the specific value of $N_{\mathrm{O}}$ is irrelevant for our purposes. If we expand the $K$ 's above for low $\Phi_{\alpha}$ values, these may assume the form of Eq. (45b) with $\widetilde{K}$ being identified as

$\widetilde{K}= \begin{cases}1 & \text { for } K=K_{1}, K_{3} ; \\ \left(1+\left(|Z|^{2}-k Z_{-}^{4}\right) / N_{\mathrm{c}}\right)^{-1} & \text { for } K=K_{2} .\end{cases}$

Compared to the $K$ 's adopted in Ref. [1], we remark that the denominator $N_{\mathrm{c}}$ in the equations above is replaced by -4 there. This is obvious since $v=1$ in Eq. (1) is associated with $N_{\mathrm{c}}=-4$ in Eq. (2).

Adapting the general formulae of Ref. [34] to the case with one hidden-sector field, as done in Ref. [1], we obtain the SSB terms in the effective low energy potential which can be written as

$V_{\mathrm{SSB}}=\widetilde{m}_{\alpha}^{2}\left|\widehat{\Phi}_{\alpha}\right|^{2}+\left(A h \widehat{\Phi}_{1} \widehat{\Phi}_{2} \widehat{\Phi}_{3}+B \mu \widehat{\Phi}_{4} \widehat{\Phi}_{5}+\right.$ h.c. $)$,

where the canonically normalized fields $\widehat{\Phi}_{\alpha}=\langle\widetilde{K}\rangle^{1 / 2} \Phi_{\alpha}$ are denoted by hats. In deriving the values of the SSB parameters above, we find it convenient to distinguish the cases:

(a) For $K=K_{1}$ and $K_{3}$, we see from Eq. (48) that $\widetilde{K}$ is constant and so the relevant derivatives are eliminated. 


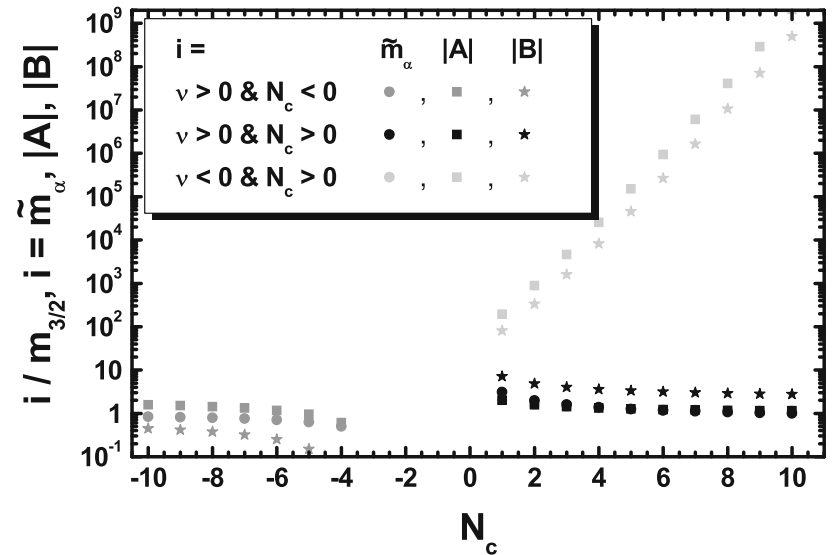

Fig. 5 The ratios $m_{\alpha} / m_{3 / 2}, A / m_{3 / 2}$ and $B / m_{3 / 2}$ for $K=K_{2}$ (circles, squares and stars respectively) versus $N_{\mathrm{c}}$. We take $N_{\mathrm{c}}<0$ (gray symbols) or $N_{\mathrm{c}}>0$ and $v>0$ (black symbols) or $N_{\mathrm{c}}>0$ and $v<0$ (light gray symbols)

Substituting

$$
\begin{aligned}
\left\langle F^{Z}\right\rangle & =\left\langle\bar{F}^{\bar{Z}}\right\rangle=\sqrt{3} m_{3 / 2} /\langle\mathrm{g}\rangle^{1 / 2} \\
\left\langle e^{K_{\mathrm{Hc}}}\right\rangle & =\langle\mathrm{g}\rangle^{-N_{\mathrm{c}} / 2},\left\langle\partial_{Z} K_{\mathrm{Hc}}\right\rangle=\frac{2 v}{\sqrt{3}}\langle\mathrm{~g}\rangle^{1 / 2}
\end{aligned}
$$

into the relevant expressions [1] we arrive at

$\tilde{m}_{\alpha}=m_{3 / 2}$ and $A=B+m_{3 / 2}=2 v m_{3 / 2} /\langle\mathrm{g}\rangle^{N_{\mathrm{c}} / 4}$.

(b) For $K=K_{2}, \widetilde{K}$ in Eq. (48) is $Z$ dependent with $\langle\widetilde{K}\rangle=\langle\mathrm{g}\rangle^{1 / 2}$ and the relevant derivatives are found to be

$$
\begin{aligned}
\left\langle\partial_{Z} \ln \widetilde{K}^{2}\right\rangle & =\frac{2}{3}\left\langle\partial_{Z} \ln \widetilde{K}^{3}\right\rangle=-\frac{4 v}{\sqrt{3} N_{\mathrm{c}}}\langle\mathrm{g}\rangle^{1 / 2}, \\
\left\langle\partial_{\bar{Z}} \partial_{Z} \ln \tilde{K}\right\rangle & =\frac{4 v^{2}}{3 N_{\mathrm{c}}^{2}}\langle\mathrm{~g}\rangle-\frac{1}{N_{\mathrm{c}}}\langle\mathrm{g}\rangle^{1 / 2} .
\end{aligned}
$$

Inserting the expressions above into the general formulae [1] we end up with

$$
\begin{aligned}
\tilde{m}_{\alpha} & =\frac{3}{4|v|} m_{3 / 2} /\langle\mathrm{g}\rangle^{1 / 2}, \\
A & =\frac{9}{8 v} m_{3 / 2} /\langle\mathrm{g}\rangle^{\left(N_{\mathrm{c}}+7\right) / 4}, \\
B & =\frac{3}{2 v}(1-v) m_{3 / 2} /\langle\mathrm{g}\rangle^{\left(N_{\mathrm{c}}+4\right) / 4},
\end{aligned}
$$

where the last result reveals the reason for which $B$ vanishes for $v=1[1]$.

The hierarchy of the SSB parameters above w.r.t $m_{3 / 2}$ is demonstrated in Fig. 5, where we display the ratios $\tilde{m}_{\alpha} / m_{3 / 2}$ (circles), $|A| / m_{3 / 2}$ (squares) and $|B| / m_{3 / 2}$ (stars) versus $N_{\mathrm{c}}$, for integer $N_{\mathrm{c}}$ values and $\left|N_{\mathrm{c}}\right| \leq 10$. We take $N_{\mathrm{c}}<0$ (gray symbols) or $N_{\mathrm{c}}>0$ and $v>0$ (black symbols) or $N_{\mathrm{c}}>0$ and $v<0$ (light gray symbols). Note that the $\tilde{m}_{\alpha}$ 's corresponding to the same $N_{\mathrm{c}}>0$ turn out to be equal for $v<0$ and $v>0$ since these are inverse proportional to $\langle\mathrm{g}\rangle^{1 / 2} v$ which is constant for the same $N_{\mathrm{c}}$, as deduced from the last relation in Eq. (23). For this reason the black circles overlap the light grays ones in the graph. We observe that in all cases the $\tilde{m}_{\alpha}$ 's are of the order of $m_{3 / 2}$ whereas $|A|$ and $|B|$ may be much larger than it for $v<0$ and $N_{\mathrm{c}}>0$. This is due to the fact that the $v=v_{-}$values, for these $N_{\mathrm{c}}$ 's, may be much larger than unity - see light gray circles in Fig. 2. Therefore, we expect that the produced sparicle spectrum will be rather heavier than $m_{3 / 2}$ in these cases. Similar conclusions can be extracted for $K=K_{1}$ too.

Let us emphasize, finally, that the presence of $k \neq 0$ in Eq. (2) not only provides mass to the $R$ axion but also breaks explicitly $U(1)_{R}$ and so, no topological defects are generated when $Z$ acquires its v.e.v in Eq. (26). Otherwise, $\langle z\rangle$ could break the discrete subgroup to which $U(1)_{R}$ is broken, due the SSB parameters in $V_{\mathrm{SSB}}$, and the production of topological defects could be possible.

\subsection{Generation of the $\mu$ term of MSSM}

Extending further the results in Ref. [1], we now check if the hidden-sector models introduced in this work offer an explanation of the $\mu$ term of MSSM, following the recipe of Guidice and Masiero in Ref. [37]. To this end, we assign the $R$ charges for the MSSM fields indicated in Ref. [1], which forbid terms of the form $H_{u} H_{d}$ in $W_{\mathrm{O}}$ - see Eq. (46). Namely, we may write it as

$W_{\mathrm{MSSM}}=h_{\alpha \beta \gamma} \Phi_{\alpha} \Phi_{\beta} \Phi_{\gamma} / 6$

where we closely follow the notation established in Ref. [1]. The mixing term between $H_{u}$ and $H_{d}$ may emerge in the part of the potential including the SSB terms

$$
\begin{aligned}
V_{\mathrm{SSB}}= & \widetilde{m}_{\alpha}^{2}\left|\widehat{\Phi}_{\alpha}\right|^{2}+\left(\frac{1}{6} A_{\alpha \beta \gamma} h_{\alpha \beta \gamma} \widehat{\Phi}_{\alpha} \widehat{\Phi}_{\beta} \widehat{\Phi}_{\gamma}\right. \\
& \left.+\widetilde{B} \mu \widehat{H}_{u} \widehat{H}_{d}+\text { h.c. }\right),
\end{aligned}
$$

if we incorporate (somehow) into the $K$ 's of Eqs. (47a) (47c) the following higher order terms, inspired by Ref. [37],

$\Delta K_{\mu}=\lambda_{\mu} \frac{\bar{Z}^{2 v}}{m_{\mathrm{P}}^{2 v}} H_{u} H_{d}+$ h.c.

where $\lambda_{\mu}$ is a real constant and the hatted fields in Eq. (55) are related to the unhatted ones as shown below Eq. (49). Due to the adopted $R$ symmetry, the terms in Eq. (56) do not coincide with those proposed in the original paper [37]. It is, therefore, non-trivial to find out the magnitude of the resulting $\widetilde{B} \mu$. 
We consider several Kähler potentials to exemplify our approach, as done in Ref. [1]. Namely, we select

$$
\begin{aligned}
K_{11}= & K_{1}+\Delta K_{\mu} \\
K_{21}= & K_{2}+\Delta K_{\mu} \\
K_{22}= & N_{\mathrm{c}} \ln \left(1+\left(|Z|^{2}-k Z_{-}^{4}+\sum_{\alpha}\left|\Phi_{\alpha}\right|^{2}+\Delta K_{\mu}\right)\right. \\
& \left./ N_{\mathrm{c}}\right) \\
K_{23}= & N_{\mathrm{c}} \ln \left(1+\left(|Z|^{2}-k Z_{-}^{4}+\Delta K_{\mu}\right) / N_{\mathrm{c}}\right) \\
& +\sum_{\alpha}\left|\Phi_{\alpha}\right|^{2}
\end{aligned}
$$

where $K_{1}$ and $K_{2}$ are defined in Eqs. (47a) and (47b) respectively. The $K$ 's above may be brought into the form

$$
K_{\mathrm{MSSM}}=K_{\mathrm{Hc}}(Z)+\widetilde{K}(Z)\left|\Phi_{\alpha}\right|^{2}+\left(C_{H} H_{u} H_{d}+\text { h.c. }\right),
$$

where $\widetilde{K}$ is determined as shown in Eq. (48). Namely the branch of the definition of $\widetilde{K}$ in Eq. (48) for $K=K_{1}$ and $K_{3}$ corresponds to $K=K_{11}$ and $K_{23}$, whereas that one which is valid for $K=K_{2}$ corresponds to $K=K_{21}$ and $K_{22}$. As a consequence, the derived $\tilde{m}_{\alpha}$ and $A_{\alpha \beta \gamma}$ in Eq. (51) remain the same for $K=K_{11}$ and $K_{23}$, whereas those shown in Eqs. (53a) and (53b) are found also for $K=K_{21}$ and $K_{22}$.

For the computation of $\widetilde{B} \mu$ in Eq. (55) it is crucial to find out $C_{H}$ in Eq. (58). This is done by expanding the $K$ 's in Eqs. (57a) - (57d) for low $H_{u}$ and $H_{d}$ values. Our result is

$C_{H}=\lambda_{\mu} \frac{\bar{Z}^{2 v}}{m_{\mathrm{P}}^{2 v}} \begin{cases}1 & \text { for } K=K_{11}, K_{21} ; \\ \left(1+\frac{|Z|^{2}-k Z_{-}^{4}}{N_{\mathrm{c}}}\right)^{-1} & \text { for } K=K_{22}, K_{23} .\end{cases}$

Thanks to the non-vanishing $C_{H}$, we expect that the effective coefficient $\widetilde{B} \mu$ in Eq. (55) assumes a non-vanishing value which may be found by applying the relevant formula in Refs. [1,34]. In particular, we obtain

$$
\frac{\widetilde{B} \mu}{m_{3 / 2}^{2}}=\lambda_{\mu}\left(\frac{4 v^{2}}{3}\right)^{v} \cdot \begin{cases}4(v-1) & \text { for } K=K_{11} \\ 3(6 v-5) / 2 v\langle\mathrm{~g}\rangle & \text { for } K=K_{21} \\ 9(1-2 v) / 8 v^{2}\langle\mathrm{~g}\rangle & \text { for } K=K_{22} \\ 3(2-3 v) / v & \text { for } K=K_{23}\end{cases}
$$

where we take into account the following:

(a) For $K=K_{11}$ and $K_{21}, C_{H}$ in Eq. (59) is only $\bar{Z}$ dependent and so we have

$$
\left\langle C_{H}\right\rangle=\left\langle\partial_{\bar{Z}} C_{H}\right\rangle / \sqrt{3}=\lambda_{\mu}\left(4 v^{2} / 3\right)^{v} .
$$

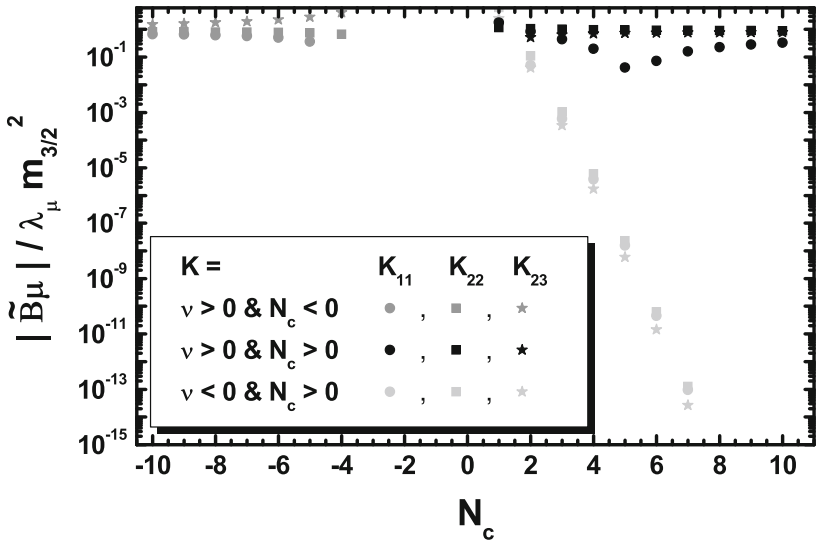

Fig. 6 The ratios $\widetilde{B} \mu / \lambda_{\mu} m_{3 / 2}^{2}$ for $K=K_{11}, K_{22}$ and $K_{23}$ (circles, squares and stars respectively) versus $N_{\mathrm{c}}$. We take $N_{\mathrm{c}}<0$ (gray symbols) or $N_{\mathrm{c}}>0$ and $v>0$ (black symbols) or $N_{\mathrm{c}}>0$ and $v<0$ (light gray symbols)

As regards $\widetilde{K}$, this is trivial for $K=K_{11}$, whereas for $K=$ $K_{21}$, its derivatives w.r.t $Z$ can be computed with the aid of Eq. (52b). Note that for $K=K_{11}$ and $v=1$ we obtain $\widetilde{B} \mu=0$ and so no $\mu$ term arises - cf. Ref. [1]. This result though is not generic.

(b) For $K=K_{22}$ and $K_{23}, C_{H}$ in Eq. (59) is both $Z$ and $\bar{Z}$ dependent and so the results in Eq. (60) can be reproduced making use of the following identities

$$
\begin{aligned}
\left\langle C_{H}\right\rangle & =-\frac{\sqrt{3} N_{\mathrm{c}}}{2 v \sqrt{\langle\mathrm{g}\rangle}}\left\langle\partial_{Z} C_{H}\right\rangle=\lambda_{\mu}\left(\frac{4 v^{2}}{3}\right)^{\nu}\langle\mathrm{g}\rangle^{1 / 2} \\
\left\langle\partial_{\bar{Z}} C_{H}\right\rangle & =\left\langle C_{H}\right\rangle\left(\sqrt{3} /\langle\mathrm{g}\rangle^{1 / 2}-2 v / \sqrt{3} N_{\mathrm{c}}\right) ; \\
\left\langle\partial_{\bar{Z}} \partial_{Z} C_{H}\right\rangle & =\frac{1}{N_{\mathrm{c}}}\left\langle C_{H}\right\rangle\langle\mathrm{g}\rangle^{1 / 2}\left(\frac{8 v^{2}}{3 N_{\mathrm{c}}}\langle\mathrm{g}\rangle^{1 / 2}-(2 v+1)\right)
\end{aligned}
$$

The derivatives of $\widetilde{K}$ w.r.t $Z$ do not vanish only for $K=K_{22}$ and are computed by employing Eq. (52b), as previously.

The magnitude of the derived $\widetilde{B} \mu$ 's above w.r.t $m_{3 / 2}^{2}$ is demonstrated in Fig. 6, where we present the ratios $\widetilde{B} \mu / \lambda_{\mu} m_{3 / 2}^{2}$ for $K=K_{11}$ (circles), $K_{22}$ (squares) and $K_{23}$ (stars) versus $N_{\mathrm{c}}$, for integer $N_{\mathrm{c}}$ values and $\left|N_{\mathrm{c}}\right| \leq 10$. We take $N_{\mathrm{c}}<0$ (gray symbols) or $N_{\mathrm{c}}>0$ and $v>0$ (black symbols) or $N_{\mathrm{c}}>0$ and $v<0$ (light gray symbols). We observe that the $\widetilde{B} \mu$ values, for $\lambda_{\mu}$ 's of order unity, are comparable to $m_{3 / 2}^{2}$ for any $v$ in the two first cases, whereas for $N_{\mathrm{c}}>0$ and $v<0$ these decrease as $N_{\mathrm{c}}$ (and $|v|$ ) increase. In his context, therefore, besides the much heavier than $m_{3 / 2}$ sfermion spectrum - see Fig. 5 - we may obtain $\mu \ll m_{3 / 2}$. The latter result is welcome, since it enhances the electroweak naturalness [39] of MSSM. 


\section{Conclusions}

We completed our approach, initiated in Ref. [1], to the problem SUSY breaking with a mildly violated $R$ symmetry. Our setting is relied on the super- and Kähler potentials given in Eqs. (1) and (2) respectively. We revealed that the solution $(v, N)=(1,-4)$ presented in Ref. [1] is just one from a class of solutions obtained by considering various values for the $R$ character of the goldstino superfield, $Z$, and letting free the geometry of the internal space. In particular, we found "magic" pairs $(\nu, N)=\left(\nu, N_{\mathrm{c}}\right)$, with $N_{\mathrm{c}}$ satisfying Eq. (15) and $v$ in the domains of Eq. (24), which allow for a naturally vanishing cosmological constant. Specific examples are presented in Table 1 and in Figs. 2 and 3 for the cases of $S U(1,1) / U(1)$ and $S U(2) / U(1)$ Kähler manifolds.

The presence of the cosmologically dangerous $R$ axion in the spectrum of the model can be eluded switching on a quartic term in the Kähler potential - i.e., setting $k \neq 0$ in Eq. (2) - which mildly breaks the $R$ symmetry without modifying the SUGRA potential, along its real direction, and the position of the Minkowski vacua in Eq. (26). The mass hierarchy between the $R$ saxion and axion and $\widetilde{G}$ is given in Eqs. (42a) and (42b) and deviates from the one found in Ref. [1]. Our scheme not only provides non-vanishing SSB (i.e., soft SUSY-breaking) parameters, independent from $k$, but also offers an explanation of the $\mu$ problem of MSSM inspired by the Giudice-Masiero mechanism. Broadening the outputs of Ref. [1] - according to which the SSB and $\mu$ parameters turn out to be of the order of $m_{3 / 2}$ - here we specify patterns of SUSY breaking which lead to values for the aforementioned parameters much larger or lower than $m_{3 / 2}$.

The crucial difference of our proposal compared to the noscale models $[2,3]$ is that here the SUSY breaking vacuum is obtained for a specific value of $Z$, whereas in those models the minimization of the SUGRA potential occurs along a $Z$-flat direction, i.e., for any values of $Z$. As a consequence, $\widetilde{G}$ mass varies with $Z$ remaining thereby undetermined, whereas it depends explicitly on $\langle z\rangle$ and the fundamental parameters $v$ and $m$ within our models. In the majority of the no-scale models the SSB masses turn out to be zero contrary to what happens in our setting.

Unfortunately, our scheme does not support viable inflationary solutions driven by $Z$ for any of the $\left(v, N_{\mathrm{c}}\right)$ values examined. Namely, the distinct values of $v$ and $N_{\mathrm{c}}$, entailed by Eqs. (15) or (16), can not be reconciled with the current inflationary data. However, the hidden sector analyzed in our work may coexist with an inflationary one provided that both sectors respect the same $R$ symmetry - for $R$-symmetry-compatible inflationary models see, e.g., Refs. [6,59-62]. The variety of the $R$ charges of $Z$ allowed within our framework is expected to facilitate the "marriage" of both sectors. For some recent attempts towards this direction see Refs. [419].

Finally, it would be interesting to investigate the lowenergy consequences of the relations between the SSB mass parameters which are usually imposed at a high scale as boundary conditions for the evolution of the relevant renormalization group equations. To implement this program we have to specify the SSB masses for gauginos besides those found in Sect. 3.1 for the scalars. As explained in Ref. [1], the safest option is the assumption that the gauginos acquire SSB masses from gauge anomalies so that the $R$ symmetry violation is conserved mild enough. This conclusion remains intact from the modifications made in the present work. The derived SUSY spectrum and the relevant cosmo-phenomenological constraints could assist us to constrain further the parameters of our models and help us to distinguish which of them is the most compelling.

Acknowledgements I would like to thank G. Lazarides for useful discussions. This research work was supported by the Hellenic Foundation for Research and Innovation (H.F.R.I.) under the "First Call for H.F.R.I. Research Projects to support Faculty members and Researchers and the procurement of high-cost research equipment grant" (Project Number: 2251).

Data Availability Statement This manuscript has no associated data or the data will not be deposited. [Authors' comment: There is no associated data to this theoretical paper.]

Open Access This article is licensed under a Creative Commons Attribution 4.0 International License, which permits use, sharing, adaptation, distribution and reproduction in any medium or format, as long as you give appropriate credit to the original author(s) and the source, provide a link to the Creative Commons licence, and indicate if changes were made. The images or other third party material in this article are included in the article's Creative Commons licence, unless indicated otherwise in a credit line to the material. If material is not included in the article's Creative Commons licence and your intended use is not permitted by statutory regulation or exceeds the permitted use, you will need to obtain permission directly from the copyright holder. To view a copy of this licence, visit http://creativecomm ons.org/licenses/by/4.0/.

Funded by $\mathrm{SCOAP}^{3}$.

\section{Appendix A: Beyond the effective superpotential}

The consideration of $W_{\mathrm{H}}$ in Eq. (1), with $v \neq 0$ a real number, as an effective superpotential offers an elegant and economical way for the presentation of our proposal. However, if we confine ourselves to positive fractional $v$ values, we can obtain a full-range, i.e. valid in (almost) the whole complex plane, superpotential.

Namely, performing a field redefinition, inspired by Ref. [47], we can show that our models can be formulated equivalently without any restriction (originated from $W_{\mathrm{H}}$ ) on the chiral superfield. Indeed, if we define

$v=p / q$ and $Z=X^{q}$, 
where $p$ and $q$ are positive integers, then $W_{\mathrm{H}}$ and $K_{\mathrm{H}}$ Eqs. (1) and (2) read

$W_{\mathrm{H}}=m X^{p}$,

which is obviously analytic in the whole complex plane, and

$K_{\mathrm{H}}=N \ln \left(1+\left(|X|^{2 q}-k\left(X^{q}-\bar{X}^{q}\right)^{4}\right) / N\right)$,

where $|X|^{2 q} \lesssim-N$ for $N<0$. The $R$ charge of $X$ is $2 / p$ and $K_{\mathrm{H}}$ violates the $R$ symmetry via the $k$-dependent term. We can easily verify that the line element $d s_{\mathrm{H}}^{2}$ in the moduli space remains unaltered in this formulation of our setting, i.e.,

$d s_{\mathrm{H}}^{2}=\partial_{X} \partial_{\bar{X}} K_{\mathrm{H}} d X d \bar{X}=\partial_{Z} \partial_{\bar{Z}} K_{\mathrm{H}} d Z d \bar{Z}$,

where $\partial_{Z} \partial_{\bar{Z}} K_{\mathrm{H}}=\mathrm{g}(Z, \bar{Z})$ is given by Eq. (5a) whereas

$\partial_{X} \partial_{\bar{X}} K_{\mathrm{H}}=q^{2}|X|^{2(q-1)} \mathrm{g}\left(Z=X^{q}, \bar{Z}=\bar{X}^{q}\right)$.

From the last expression we deduce that the $X-\bar{X}$ metric or, equivalently, the kinetic term in the $X-\bar{X}$ Lagrangian is well defined for $q \geq 1$.

Repeating the analysis of Sect. 2.2.1 we can easily assure that the condition in Eq. (15), which now takes the form

$N_{\mathrm{c}}=4 p^{2} / q(3 q-4 p)$,

allows for a SUSY-breaking Minkowski vacuum

$$
\langle|X|\rangle=\left(\frac{2 p}{\sqrt{3} q}\right)^{1 / q} \text { with } \operatorname{Im} X=0 \text { and } \frac{p}{q} \neq \frac{3}{2}
$$

- cf. Eqs. (20) and (26). Some solutions to Eq. (A.6) are arranged in the two upper lines of Table 1. However, the present form of the condition in Eq. (A.6) includes redundant solutions when $p$ and $q$ are not relatively prime numbers. If we derive the particle spectrum at the vacuum of Eq. (A.7), we can verify that this is identical with that exposed in Secs. 2.2.3 and 2.3 replacing $v=p / q$. As a consequence, the supertrace relation in Eq. (40) remains also intact. Finally, the redefinitions in Eq. (A.1) let unaffected the derivation of the SSB parameters in Sect. 3.

Therefore, our construction is valid not only for the effective $W_{\mathrm{H}}$ in Eq. (1) but also for the exact $W_{\mathrm{H}}$ in Eq. (A.2) in conjunction with $K_{\mathrm{H}}$ in Eq. (2) or Eq. (A.3) correspondingly.

\section{References}

1. C. Pallis, Phys. Rev. D 100(5), 055013 (2019). arXiv:1812.10284

2. J. Ellis, B. Nagaraj, D.V. Nanopoulos, K.A. Olive, J. High Energy Phys. 11, 110 (2018). arXiv: 1809.10114

3. J. Ellis, B. Nagaraj, D.V. Nanopoulos, K.A. Olive, S. Verner, J. High Energy Phys. 10, 161 (2019). arXiv:1907.09123

4. J. Ellis, D.V. Nanopoulos, K.A. Olive, S. Verner, Phys. Rev. D 100(2), 025009 (2019). arXiv:1903.05267
5. J. Ellis, D.V. Nanopoulos, K.A. Olive, S. Verner, J. Cosmol. Astropart. Phys. 09, 040 (2019). arXiv:1906.10176, arXiv:2004.00643

6. I. Antoniadis, A. Chatrabhuti, H. Isono, R. Knoops, Eur. Phys. J. C 79(7), 624 (2019). arXiv:1905.00706

7. W. Buchmuller, L. Covi, D. Delepine, Phys. Lett. B 491, 183 (2000). arXiv:hep-ph/0006168

8. W. Buchmüller, E. Dudas, L. Heurtier, C. Wieck, J. High Energy Phys. 14, 053 (2014). arXiv:1407.0253

9. E. Dudas, T. Gherghetta, Y. Mambrini, K.A. Olive, Phys. Rev. D 96(11), 115032 (2017). arXiv: 1710.07341

10. I. Dalianis, F. Farakos, A. Kehagias, A. Riotto, R. von Unge, J. High Energy Phys. 01, 043 (2015). arXiv:1409.8299

11. K. Schmitz, T.T. Yanagida, Phys. Rev. D 94(7), 074021 (2016). arXiv:1604.04911

12. V. Domcke, K. Schmitz, Phys. Rev. D 95(7), 075020 (2017). arXiv: 1702.02173

13. V. Domcke, K. Schmitz, Phys. Rev. D 97(11), 115025 (2018). arXiv: 1712.08121

14. M.C. Romão, S.F. King, J. High Energy Phys. 07, 033 (2017). arXiv: 1703.08333

15. S.F. King, E. Perdomo, J. High Energy Phys. 05, 211 (2019). arXiv: 1903.08448

16. R. Kallosh, A. Linde, Phys. Rev. D 91, 083528 (2015). arXiv: 1502.07733

17. A. Linde, J. Cosmol. Astropart. Phys. 11, 002 (2016). arXiv: 1608.00119

18. Y. Aldabergenov, S.V. Ketov, Phys. Lett. B 761, 115 (2016). arXiv: 1607.05366

19. Y. Aldabergenov, A. Chatrabhuti, S.V. Ketov, Eur. Phys. J. C 79(8), 713 (2019). arXiv: 1907.10373

20. C.D. Froggatt, R. Nevzorov, H.B. Nielsen, A.W. Thomas, Int. J. Mod. Phys. A 35(01), 2050007 (2020). arXiv:1909.02124

21. Y. Aldabergenov, Phys. Rev. D 101, 015016 (2020). arXiv: 1911.07512

22. C.P. Burgess et al., Fortsch. Phys. 68(10), 2000076 (2020). arXiv:2006.06694

23. H.P. Nilles, Phys. Rep. 110, 1 (1984)

24. U.H. Danielsson, T. Van Riet, Int. J. Mod. Phys. D 27(12), 1830007 (2018). arXiv:1804.01120

25. R. Kallosh, T. Wrase, Fortsch. Phys. 67(1-2), 1800071 (2019). arXiv: 1808.09427

26. J. Polonyi, Budapest preprint KFKI/1977/93 (1977)

27. M. Claudson, L. Hall, I. Hinchliffe, Phys. Lett. B 130, 260 (1983)

28. C. Pallis, N. Toumbas, J. Cosmol. Astropart. Phys. 05(05), 015 (2016). arXiv: 1512.05657

29. C. Panagiotakopoulos, Phys. Rev. D 71, 063516 (2005). arXiv:hep-ph/0411143

30. G.K. Chakravarty, G. Gupta, G. Lambiase, S. Mohanty, Phys. Lett. B 760, 263 (2016). arXiv:1604.02556

31. G.K. Chakravarty, U.K. Dey, G. Lambiase, S. Mohanty, Phys. Lett. B 763, 501 (2016). arXiv:1607.06904

32. J.J.M. Carrasco, R. Kallosh, A. Linde, D. Roest, Phys. Rev. D 92(5), 041301 (2015). arXiv: 1504.05557

33. A.E. Nelson, N. Seiberg, Nucl. Phys. B 416, 46 (1994). arXiv:hep-ph/9309299

34. A. Brignole, L.E. Ibáñez, C. Muñoz, Adv. Ser. Direct. High Energy Phys. 18, 125 (1998). arXiv:hep-ph/9707209

35. V. Kaplunovsky, J. Louis, Nucl. Phys. B 444, 191 (1995). arXiv:hep-th/9502077

36. K. Choi, H.P. Nilles, J. High Energy Phys. 04, 006 (2007). arXiv:hep-ph/0702146

37. G.F. Giudice, A. Masiero, Phys. Lett. B 206, 480 (1988)

38. K.J. Bae, H. Baer, V. Barger, D. Sengupta, Phys. Rev. D 99(11), 115027 (2019). arXiv: 1902.10748 
39. H. Baer et al., Eur. Phys. J. ST 229(21), 3085 (2020). arXiv:2002.03013

40. J. Jaeckel, M. Spannowsky, Phys. Lett. B 753, 482 (2016). arXiv: 1509.00476

41. J.S. Lee, arXiv: 1808.10136

42. P.F. Depta, M. Hufnagel, K. Schmidt-Hoberg, J. Cosmol. Astropart. Phys. 05, 009 (2020). arXiv:2002.08370

43. F. Ertas, F. Kahlhoefer, J. High Energy Phys. 07, 050 (2020). arXiv:2004.01193

44. G. Lucente et al., J. Cosmol. Astropart. Phys. 12, 008 (2020). arXiv:2008.04918

45. D. Castaño, D.Z. Freedman, C. Manuel, Nucl. Phys. B 461, 50 (1996). arXiv:hep-ph/9507397

46. P. Binetruy, Phys. Rev. D 60, 063502 (1999). arXiv:hep-ph/9810553

47. X. Gao, T. Li, P. Shukla, Phys. Lett. B 738, 412 (2014). arXiv: 1404.5230

48. I. Affleck, M. Dine, N. Seiberg, Nucl. Phys. B 241, 493 (1984)

49. G. 't Hooft, NATO Sci. Ser. B 59, 135 (1980)

50. L.E. Ibáñez, D. Lüst, Nucl. Phys. B 382, 305 (1992). arXiv:hep-th/9202046

51. D. Lüst, S. Reffert, S. Stieberger, Nucl. Phys. B 727, 264 (2005). arXiv:hep-th/0410074
52. G. 't Hooft, Phys. Rev. Lett. 37, 8 (1976)

53. H.S. Goh, M. Ibe, J. High Energy Phys. 03, 049 (2009). arXiv:0810.5773

54. Y. Hamada et al., J. Cosmol. Astropart. Phys. 01, 024 (2014). arXiv: 1310.0118

55. P. Baratella et al., J. High Energy Phys. 06, 086 (2016). arXiv:1603.05682

56. D. Bardhan et al., J. High Energy Phys. 06, 129 (2016). arXiv: 1603.05251

57. A.H. Chamseddine, H. Dreiner, Nucl. Phys. B 458, 65 (1996). arXiv:hep-ph/9504337

58. S. Ferrara, R. Kallosh, A. Linde, J. High Energy Phys. 10, 143 (2014). arXiv:1408.4096

59. C. Pallis, Q. Shafi, Phys. Lett. B 725, 327 (2013). arXiv: 1304.5202

60. C. Pallis, J. Cosmol. Astropart. Phys. 10, 058 (2014). arXiv: $1407.8522 \mathrm{D}$

61. C. Pallis, Phys. Rev. D 92(12), 121305(R) (2015). arXiv: 1511.01456

62. C. Pallis, Universe 4(1), 13 (2018). arXiv:1710.05759 